\title{
Improving the method for determining the granular media strength performance by oblique shear test
}

\author{
Sergey Shabaev ${ }^{1, *}$, Nikita Martel $^{1}$, Artemy Shtark ${ }^{1}$ and Elmira Rysbekova ${ }^{2}$ \\ ${ }^{1}$ T.F. Gorbachev Kuzbass State Technical University, 28 Vesennyayastreet, Kemerovo, Russian \\ Federation \\ ${ }^{2}$ M.M. Adyshev Osh Technological University, 81 N. Isanovstreet, Osh, Kyrgyz Republic
}

\begin{abstract}
To ensure the strength performance of the pavement of open pit truck roads, it is necessary that it conforms not only to the designed required deformation (elasticity) modulus, but also to the condition for ensuring the shear resistance of structural layers made from non-cohesive and lowly cohesive granular media. However, today there are no reliable methods for determining the strength performance of coarse-grained materials, therefore, for them there are no designstrength values, and therefore their shear resistance is not evaluated, which is a serious omission. It is proposed to evaluate the strength performance of granular media by the oblique sheartest, for which an improved design of oblique shear apparatus was developed, which made it possible to adjust the granular medium density (structure) to the corresponding density (structure) under construction conditions. An analysis was also made of the influence of the angle of inclination of the shear area on the predicted values of normal and shear stresses arising in the cross section, which made it possible to establish the recommended values of this indicator depending on the prevailing particle size of the test medium.
\end{abstract}

\section{Introduction}

In opencast and quarry mining, one of the most important technological operations is the rock mass haulage, which is carried out mainly using motor vehicles [1]. And, despite the fact that open pit haul roads with pavement made of layers of asphalt concrete and geosynthetic materials are provided for the possibility and efficiency of the use of dump trucks [2-4], granular media in the form of sorted or unsorted crushed stone, including dry bound macadam, are most widely used [5].

In [6], it is noted that pavement has strength if its deformation characteristic corresponds to the design required deformation modulus. However, from the results of the pavement design, it is known that the pavement strength is consideredas the ability to resist the propagation of residual deformations and fractures under the action of tangential and normal stresses arising in structural layers and underlying subgrade from design load. Moreover, the methods for assessing structural strength includes, among others, strength

*Corresponding author: shabaev81@rambler.ru 
assessment taking into account stresses arising in individual structural layers. For structural layers of non-cohesive and lowly cohesive materials, which include granular media, strength is evaluated by the criterion of the shear resistance of materials to tangential stresses arising in them. Thus, the strength of the structure will be ensured only if the design active shear stress does not exceed the limit value of the active shear stress.

At the same time, the design of pavement strength under the condition of shear stability of non-cohesive and lowly cohesive structural layers of pavement is carried out only for underlying soils and sands, and the base layers and toppings are made from coarse-grained materials (crushed stone, gravel, crushed-sand or gravel-sand mixtures, coarse-grained soils, fragmented rocks) are conditionally accepted as rigid and shear-resistant. This convention was adopted primarily due to the fact that there are no reliable methods for determining the strength performanceof such granular media, which, of course, is a serious omission.

Today, there are various methods for determining the strength performance of granular media: direct shear, triaxial system, four-blade shear, field loading, oblique shear, shear of ground pillars. However, almost all of them are designed to determine the strength performance of dusty clay and sandy soils. In fact, the methods of direct shear, triaxial system, and oblique shear can be used to determine the strength performanceof coarsegrained media; however, the main problems are the need to use largerholders in order to avoid the wall effect [7-10], as well as ensuring the medium compaction degree during testing of the corresponding construction conditions [11-12].

\section{Materials and methods}

To determine the strength performance of granular media containing in their composition particles larger than $5 \mathrm{~mm}$ without restrictions on mass (volume), the oblique shear apparatuswas improved. For this purpose, the following changes were made in the oblique shear apparatus, which includes an axial pressure screw, an arm, a compression dynamometer, an adapter, an upper holder, a lower holder and a base carriage:

- an additional load is provided, providing vertical pressure on the granular medium of 40 $\mathrm{g} / \mathrm{cm}^{2}$. This allows you compacting the test medium either on a standard shaking table with an oscillation amplitude of $0.40 \pm 0.05 \mathrm{~mm}$ and an oscillation frequency of $48 \pm 3 \mathrm{~Hz}$, or on a hydraulic press under a pressure of $20 \mathrm{MPa}$, or any other to achieve the granular medium density (structure) corresponding to density (structure) in a natural conditions (at a construction site);

- an additional compacting device is provided, consisting of two hollow half-cylinders with stiffeners and connecting plates, which allows rigidly fixing the upper and lower holders relative to each other during compaction of the test medium in order to prevent their displacement;

- a bottom is additionally provided, which prevents the free flow of the test medium from the holders during their movement from a standard shaking or press table onto the base carriage under the axial pressure screw.

Thus, the manufactured oblique shear apparatus(Fig. 1) consists of an axial pressure screw 1 , an arm 4, a compression dynamometer 15 , an adapter 7 , a load 9 , an upper holder 5 , a lower holder 6 , a compacting device 8 , a bottom 2 , and a base carriage 3 . The arm 4 is rigidly connected to the frame 10 , the half-cylinders of the compacting device 8 are interconnected by means of a bolt connection $11,13,16$, to ensure the required clearance between the upper and lower holders; adjusting screws 12, 14 are provided. The inner diameter of the holders $d_{\text {hold }}$ is assigned to be not less than $5 \ldots 7$ times the size of the largest particle of the granular medium in order to avoid the wall effect. The total working height of the holders in the assembled state $h_{\text {work }}$ is determined by the condition of providing an 
additional distance $h_{a d}$ between the upper boundary of the upper holder 5 and the lower boundary of the lower holder 6 with a cut line located at an angle $\alpha$ of at least $40 \ldots 50 \mathrm{~mm}$. The height of the compacting device $h_{c d}$ is assigned according to the condition of complete overlap of the cut line. The height of the apparatus $h_{\text {ins }}$ should allow free placement of the base carriage 3, bottom 2, upper 5 and lower 6 holder, load 9, adapter 7 and compression dynamometer 15 under the axial pressure screw 1 with a clearance $h_{\text {res }}$ between the compression dynamometer 15 and the lower face of the arm 4 not less than $10 \ldots 20 \mathrm{~mm}$. The diameter of the load 9 is taken $0.2 \ldots 1 \mathrm{~mm}$ less than the inner diameter of the holders $d_{\text {hold }}$.

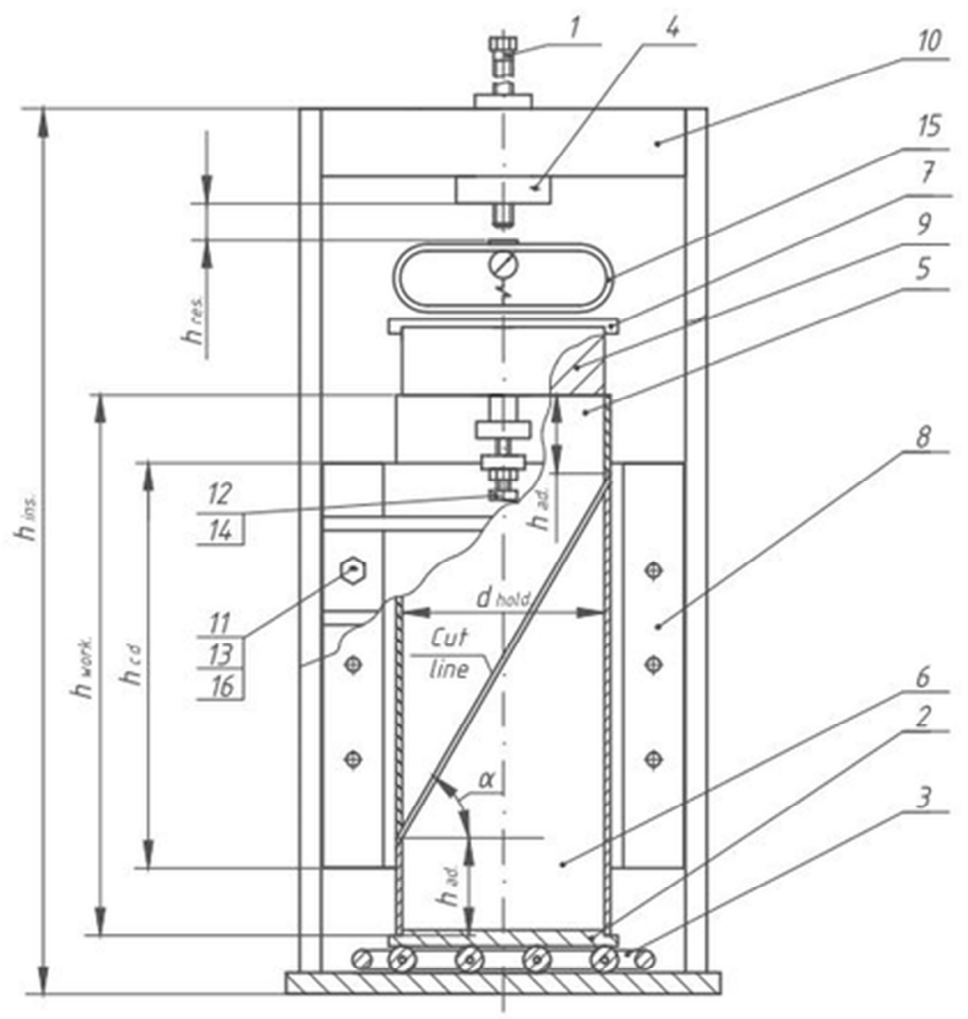

Fig. 1. Advanced oblique shear apparatus diagram.

When testing granular media, work with an improved oblique shear apparatusis as follows. The lower holder 6 is installed on the bottom 2, after which two hollow halfcylinders of the compacting device 8 , which are fastened with bolt connections $11,13,16$ without tightening, are placed upon it; then the upper holder 5 is installed and the required clearance is provided between the adjusting screws 12, 14 the upper 5 and lower 6 holders. Next, the bolt connections $11,13,16$ are tightened, the test granular medium is poured into the holders to the upper face of the upper holder, after which load 9 is placed on it and the entire assembled unit is putonto standard shaking table providing the amplitude oscillations of $0.40 \pm 0.05 \mathrm{~mm}$ and the frequency oscillation of $48 \pm 3 \mathrm{~Hz}$, or onto press plate. The granular material is compacted on a standard shaking table or on a press for 3 minutes, after which the entire assembled unit is placed on the base carriage 3, located on the base of the frame 10 , under the axial pressure screw 1 , the half-cylinders of the compacting device 8 are removed, an adapter 7 is installed on the load 9 and the compression dynamometer 15 
and the axial pressure screw 1 is brought by rotation into contact with the compression dynamometer 15 .

The sample shear is carried by the pressure created by the axial pressure screw 1 through the compression dynamometer 15, which fixes the magnitude of this force transmitted by steps equal to $10 \ldots 20$ kilogram-force. Each stage is maintained until conditional stabilization. The shear moment is fixed by rotating the arrow of the indicator inversely related to load increase. When implementing the oblique sheartest, the diagram shown in Fig. 2. From this diagram it is clear that the normal and shear stresses arising along the shear area are equal to:

$$
\begin{gathered}
\sigma=\frac{4 \cdot F \cdot \cos ^{2} \alpha}{\pi \cdot d^{2}}, \\
\tau=\frac{4 \cdot F \cdot \sin \alpha \cdot \cos \alpha}{\pi \cdot d^{2}} .
\end{gathered}
$$

where $F$ - forceresultingfromexternalload; $\alpha$ - angle of inclination of the cut line (shear area) relative to the horizontal plane.

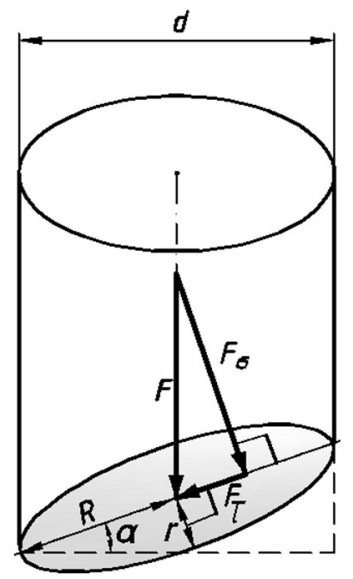

Fig. 2. The design diagram for the oblique shear test.

\section{Results and discussion}

In accordance with the well-known procedure for testing a granular medium testing, it is necessary to conduct tests at least three different values of the angle of inclination of the shear area. The standard angles are: $30^{\circ}, 40^{\circ}, 50^{\circ}$, and $60^{\circ}$. However, if we analyze the dependences of the change in the arising shear stresses on the normal stresses acting on the shear area located at different angles $\alpha$ and compare them with the ultimate values of the shear resistance of granular media at different values of the angle of internal friction $\varphi$ (specific adhesion is conventional), then the following conclusions can be drawn (Fig. 3): 


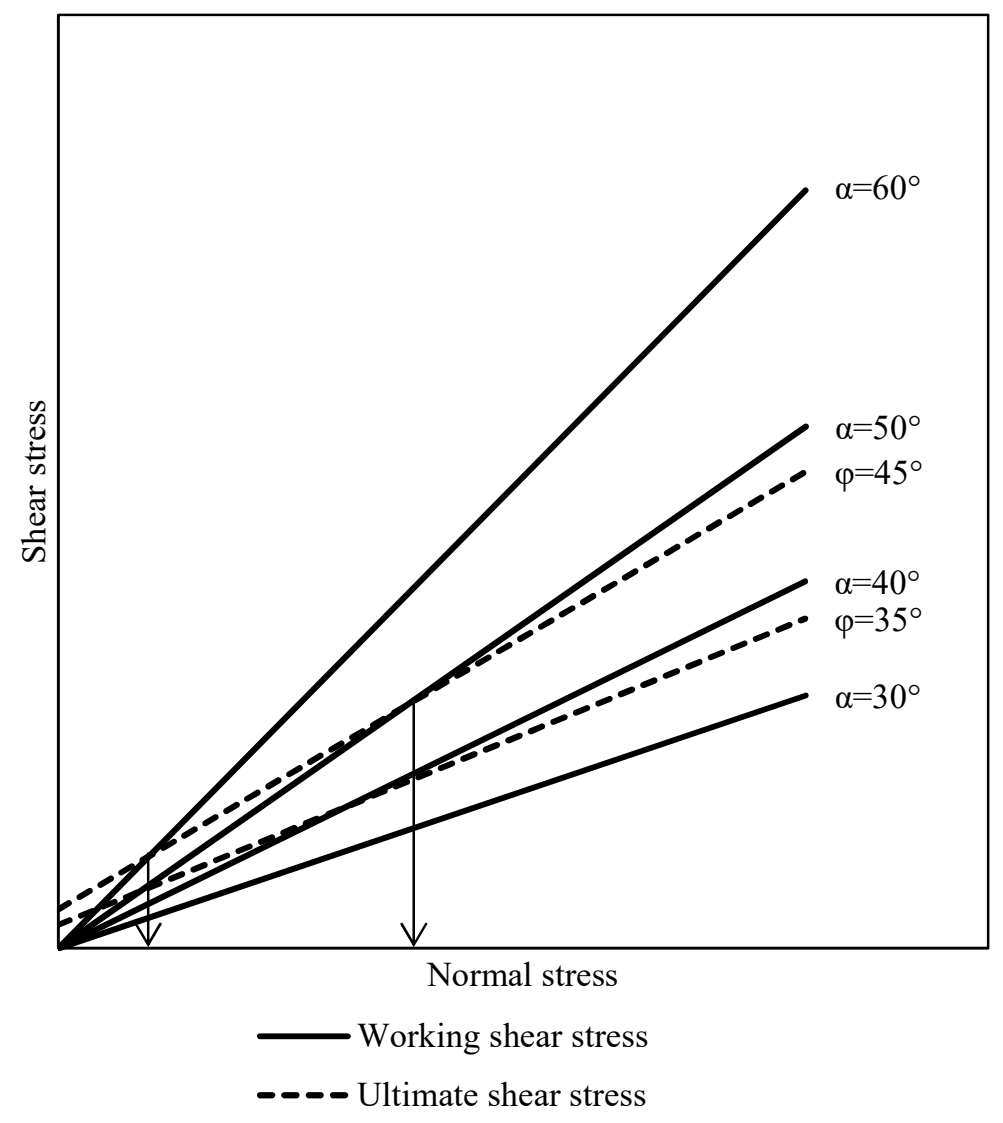

Fig. 3. Dependences of shear stresses on the magnitude of normal stresses arising along a shear area located at different angles $\alpha$ and ultimate granular media shear stresses with different angles of internal friction $\varphi$.

1. Since in most granular media the angle of internal friction is in the range from 30 to $45^{\circ}$, and in the absence or small number of particles less than $1 \mathrm{~mm}$, as a rule, exceeds $40^{\circ}$, then at angles of inclination of the shear area $\alpha=30^{\circ}$ and $\alpha=40^{\circ}$, it is not recommended to test such media, since the line of growth of the ultimate shear stresses cannot cross the lines of the working shear stresses arising along the shear area from the external load. In this case, shear is possible only when the granular media is destroyed (crushed) to a state in which its angle of internal friction decreases to a value corresponding to the angle of inclination of the shear area.

2. If you test a granular medium at angles of inclination of the shear are $\alpha=50^{\circ}$ and $\alpha=$ $60^{\circ}$, then two points will not be enough to reliably determine the desired strength performance. In this regard, it is necessary to conduct tests at intermediate angles $\alpha=45^{\circ}$ and $\alpha=55^{\circ}$.

3. When the value of the angle of internal friction of the granular media is significantly different from the angle of inclination of the shear area, the line of growth of the ultimate shear stresses intersects the line of the working shear stresses that arise along the shear area from the external load, at insignificant normal stresses. This can lead to the fact that the obtained point will not lie on the line described by Coulomb's law, since it is known that the initial section of the shear curve is non-linear for low values of the normal stress. 
Preliminary laboratory experiments showed that at an angle of inclination of the shear area $\alpha=60^{\circ}$, regardless of the size of the particles of the granular medium (there was no dusty clay fraction), shear occurs due to its own weight, and therefore this angle of inclination of the shear area can only be assigned for granular media having both a large angle of internal friction and significant adhesion. This will contribute to the shift of the point of intersection of the line of shear stress occurring at the area with the line of ultimate granular medium resistance to shear to the right, that is, the shift will occur at high normal stresses. At the same time, from Fig. 3 it is clear that the shift, if it occurs, will occur at small normal stresses, and this causes the danger of an experimental point lying outside the straight line described by Coulomb's law. Thus, this angle is recommended to be excluded from the experiment.

The experimental data also show that at an angle of inclination of the shear area $\alpha=50^{\circ}$, granular media consisting of particles smaller than $2 \mathrm{~mm}$ are displaced at a small normal stress, that is, there is a high probability that the obtained experimental data cannot be reliably described by the Coulomb's law.

Thus, taking into account the fact that according to the results of the experiment there should be at least three points obtained at different angles of inclination of the shear area, the angle of internal friction of granular media is often more than $40^{\circ}$ and, in the absence or low content of dusty clay particles, possess small adhesion; when implementing this method, it is necessary to have holders that allow testing granular media at intermediate angles of inclination of the shear area. Recommended angles of inclination of the shear area depending on the prevailing particle size of the granular medium are given in table.

Table. Recommended values of angles of inclination of the shear area when implementing the oblique shear test.

\begin{tabular}{|c|c|c|c|}
\hline $\begin{array}{c}\text { Prevailing particle } \\
\text { size of the granular } \\
\text { medium, mm }\end{array}$ & $\begin{array}{c}\text { Minimumangle of } \\
\text { inclination of the shear } \\
\text { area, degrees }\end{array}$ & $\begin{array}{c}\text { Main angle of } \\
\text { inclination of the shear } \\
\text { area, degrees }\end{array}$ & $\begin{array}{c}\text { Maximumangle of } \\
\text { inclination of the } \\
\text { shear area, degrees }\end{array}$ \\
\hline from 0,5 to1.5 & 40.0 & 42.5 & 45.0 \\
\hline from 1.5to5.0 & 42.5 & 45.0 & 47.5 \\
\hline from5.0to13.0 & 45.0 & 47.5 & 50.0 \\
\hline beyond13.0 & 47.5 & 50.0 & 52.5 \\
\hline
\end{tabular}

During the experiment, it is necessary to determine the maximum value of the vertical force that the granular medium is able to withstand at the minimum, main, and maximum angle of inclination of the shear area, after which the corresponding normal and shear stress are determined by formulas (1) and (2). After this, the granular medium strength certificate is built, the general view of which is shown in Fig. 4, on the basis of which the angle of internal friction and the adhesion of the granular medium are determined. 


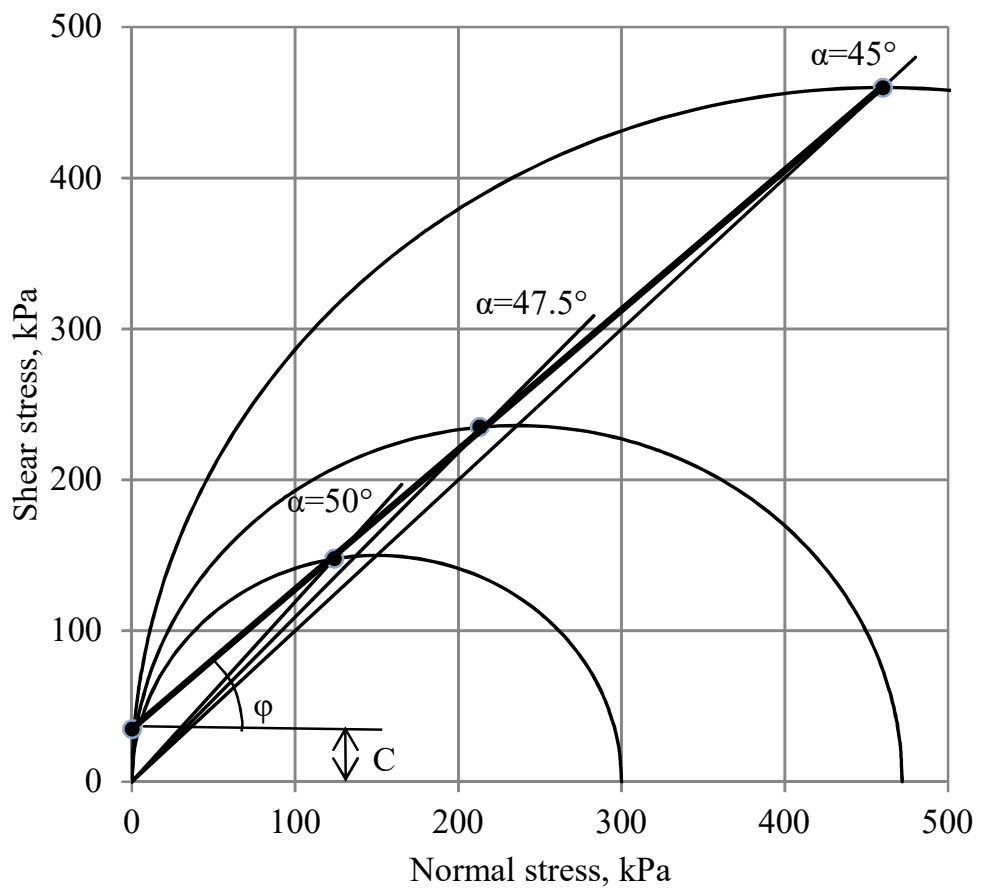

Fig. 4. An example of a granular medium strength certificate, built up according to the results of theoblique shear test.

\section{Conclusions}

1. The proposed improved laboratory wedge installation design, which additionally provides a load, compacting device and bottom, allows determining the strength performance of granular media containing particles of any size without limitation on their content. This is achieved due to the possibility of achieving the granular medium density (structure) corresponding to the density (structure) in a natural conditions (at a construction site) by compaction on a shaking table or a press.

2. It is proved that the standard values of the angle of inclination of the shear area of $30^{\circ}$, $40^{\circ}, 50^{\circ}$ and $60^{\circ}$, implemented using the oblique shear test, are not suitable for determining the strength performance of granular media, consisting mainly of particles larger than 0.5 mm.

3. It has been found that the recommended values of the angle of inclination of the shear area, implemented using the oblique shear test, depend on the prevailing particle size of the granular medium and range from $42.5^{\circ}$ to $55^{\circ}$.

\section{References}

1. P. L. Mariev, A. A. Kuleshov, A. N. Egorov, I. V. Zyrayanov, Dumptrucks: status and prospects (EDPSciences, St. Petersburg, 2004)

2. A. A. Khristoforova, M. D. Sokolova, S. E. Filippov, S. M. Semenova, I. N. Gogolev, Chemical technologies and nanomaterials, 41, 78 (2014)

3. A. A. Khristoforova, M. D. Sokolova, B. N. Zarovnyaev, A. N. Akishev, Mining Journal, 3, 47 (2016) 
4. G. V. Solovyev, Procedia Engineering, 189, 666 (2017)

5. S. A. Arefiev, Natural and technical sciences, 1, 38 (2015)

6. Yu. I. Lel, Bulletin of the Ural State Mining University, 3, 70 (2016)

7. G. E. Mueller, Powder Technology, 203, 626(2010)

8. M. M. Roozbahani, Powder Technology, 224, 46(2012)

9. C. M. Wensrich, Powder Technology, 219, 118 (2012)

10. V. G. Bondarev, Modern trends in the development of science and technology, 2-1, 12 (2015)

11. D. V. Moldovan, Journal of Industrial Pollution Control, 33-1, 1007 (2017)

12. S. L. Jackson, Geoforum, 66, 94 (2015) 